\title{
VIRTUAL PERSONALISED CHANNELS: VIDEO CONDUCTING OF FUTURE TV BROADCASTING
}

\author{
Carmen Mac Williams, Richard Wages, Stefan M. Grünvogel, Georg Trogemann \\ NOMADS Lab, Piusstr. 40, D-50823 Cologne, Germany. \\ \{macwilliams, wages, gruenvogel\}@nomadslab.org, trogemann@khm.de
}

Keywords: Video Conductor, Live Staging, Semantic Annotation, Personalisation, Intelligent Video Objects

\begin{abstract}
Television is undergoing a historical change. Interactive Digital Broadcasting will be reality in 2010+. Heaps of video material will be produced by TV broadcasters, which will overwhelm both the producers as well as the consumers. Current TV formats and forms of broadcasting do not satisfy the personal moods and interests of the consumer. We hence propose the development of a TV environment which allows for the establishment of 'virtual personalised channels'. To do so, (live) semantic annotation of video material as well as methods for live staging of media events have to be designed. The resulting drastically different process of content production and consuming will lead to the satisfaction of individual human needs.

The approaches outlined in this extended abstract are the basis for our upcoming IST research project LIVE.
\end{abstract}

\section{Introduction}

Innovations in hardware and network technologies lead to an exploding number of non-interrelated parallel media streams. Per se this means no additional value for the consumer. Broadcasting and advertisement industries have not yet found new formats to reach the individual user with their content. Television originated from theatre and film. Both media tend to be of a primarily entertaining nature and entail a lean-back mentality of the consumer [3]. To expect that consumers will simply switch to a complete lean-forward mentality - like they do when sitting in front of a PC - once iTV becomes reality is an illusion [1]. Moreover it is not desirably either, since both, producer and end user, are overwhelmed by the mere quantity of live video material provided.

\section{Current TV Industry Approaches}

Due to the growing technical possibilities the today broadcasting industry is able to offer a huge number of different channels. Nevertheless we can observe two main tendencies due to the ' 24 hour per day restriction' for every channel:

- Channels serving and restricting themselves to a singular and very special category, e.g. Golf, news loops, cartoons, region etc. (i.e. 'Greatest Common Divider' approach)

- Channels serving a mean set of interests trying to reach the mass audience (i.e. 'Least Common Denominator' approach)

Of course there are some exceptions to these tendencies. Broadcasters like some public stations try to combine broad overview with intermittent in depth coverage (e.g. arte theme night). But the current setting of autonomous media streams (i.e. 'channels') will not lead to a satisfaction of the individual consumer's needs. And obviously the number of channels serving certain moods are (and will always be) too small compared to billions of individual moods. A 'solution', to offer one channel per single consumer, again is an illusory vision (too many consumers, too few 'classical producers'). In addition each single end user has varying (and unknown) moods. To offer 'video on demand' (mainly feature films) is just a fragmentary answer to this problem since the hunger of being part of the world community and the coverage of live events cannot be satisfied. Hence solutions are urgently needed for the treatment of such live events for future digital broadcasting.

\section{Staging and Virtual Personalised Channels}

Putting the consumer in the centre of our research we ask ourselves how we can create an environment which allows for personalised TV in covering mass media events.

Our approach to this dilemma is to offer 'everything' (i.e. all, possibly unedited camera streams) and at the same time provide the prerequisites for the establishment of 'virtual personalised channels'. To do so we have to realise the following:

1) Production methods and tools for 'staging' or 'conducting' a live event have to be conceived. Design principles for staging live media events and for content creation have to be developed and workflows have to be defined which support the collaborative staging by professional users. The interfaces have to hide the underlying complexity of the media objects and the workflow requirements.

2) Personal user interests have to be linked semantically with video content. Within a robust framework, that allows for the live staging of media events, methodologies for staging and content research, content oriented detection, extraction and 
annotation of video material and the personalisation of the users [2] have to work together and enforce each other in an intelligent way. The main effort will be to develop an open framework for broadcasting environments, which utilises the interaction of cognition based content knowledge with social based consumer knowledge (connect item based knowledge with user based knowledge). Scientific questions to be answered will be:

- How/what will be annotated?

- How do we link the annotated video objects (consumer/ video conductor)

The goals of this research theme are to develop new methods, tools and interfaces for detection, extraction (of knowledge) and annotation of video material and other resources. A special focus is the 'in-vivo generation of knowledge', i.e. annotation metadata is created while content is created. More specifically this will include

- Automatic offline and online detection and extraction of knowledge from media archives and from live produced media and the annotation of media objects

- Semi-automatic online annotation of live video material. Human annotation with meta-knowledge about content will be used for constellations where automation is not possible (e.g. social meaning).

- Automatic integration of knowledge and content from external resources

- Integration of all the above methods to support the staging process

\section{Closing the Loop for Future TV Work Flow}

Equipping the audio-visual component of an intelligent video object with an additional component which holds (invisible) semantic metadata radically changes the process dynamics of producing, broadcasting and consuming. Now all essential prerequisites are given for a more 'evolutionary' character of the production process:

Instead of broadcasting just a single, carefully edited video stream chosen by a producer, several (or even all) camera streams are broadcasted. Beside the mere increase of the number of 'allowed' images this means the availability of an entire set of images for a particular incident. The variability of these (possibly imperfect) images allows for a direct or indirect (e.g. mood based) selection and a recombination not only by the professional producer, but also by the individual consumer.

As a consequence new and 'personalised' perspectives (or formats) evolve as an additional value for consumers. Closing the system by a feedback of consumer desires (e.g. annotation information) to the on-site producers (or even camera men) allows for a replication loop through the consumer's influence on the generation of new images.
This gives rise to the emergence of completely novel professions such as video conductor as an analogue to a music conductor or disk jockey who stages live events for an audience.

\section{Vision}

Our vision is, that the viewer of tomorrow will not follow channels anymore. Rather individual consumers will follow their favourite video conductor or even navigate by themselves through a mass media event such as the Olympic Games. Sometimes the viewer will be in the mood to lean back on his sofa and be guided by the video conductor, then she/he leans forward and takes control over the journey through the video streams. Thus we will serve this basic human need of loosing control (being entertained, typical couch potato behaviour) as well as taking over the control (inform her/himself, typically PC behaviour).

\section{Acknowledgements}

The LIVE project proposal (No. 27312) was submitted within the 4th Call of the 6th Framework in the IST Strategic Objective 2.4.7 'Semantic-based Knowledge and Content Systems' and will be a joint research and development effort of the following academic and commercial institutions: Fraunhofer Institute for Media Communication, Academy of Media Arts Cologne, University of Applied Sciences Cologne, Austrian Broadcasting ORF, Atos Origin s.a.e., School of Informatics at the University of Bradford, University of Ljubljana, Salzburg Research Forschungsgesellschaft m.b.H. and Sony NetServices.

\section{References}

[1] J. Freeman, J. Lessiter. "Using attitude based segmentation to better understand viewers' usability issues with Digital and Interactive TV", in Judith Masthoff, Richard Griffiths \& Lyn Pemberton (Eds.) Proceedings of EuroITV03, Brighton, UK, (2003).

[2] Hee-K. Lee, Han-K. Lee, J. Nam, B. Bae, M. Kim, K. Kang, J. Kim. "Personalized Contents Guide and Browsing based on User Preference", Proceedings of the AH2002 Workshop on Personalization in Future TV, Malaga, Spain, (2002).

[3] D. J. Melinger. "Massive Media", Thesis, Interactive Telecommunications Program, Tisch School of the Arts, New York University, http://massive-media.org/docs/mm.pdf, (2004). 